\title{
Evaluación de la percepción estudiantil en relación al uso de la plataforma Moodle desde la perspectiva del TAM
}

\section{Evaluation of the student perception in relation to the use of the Moodle platform from the TAM perspective}

\author{
Norka Bedregal-Alpaca ${ }^{1 *} \quad$ Víctor Cornejo-Aparicio ${ }^{2}$ \\ Doris Tupacyupanqui-Jaén $\quad$ Sidanelia Flores-Silva ${ }^{4}$ \\ Recibido 08 de mayo de 2019, Aceptado 14 de junio de 2019 \\ Received: May 08, 2019 Accepted: June 14, 2019
}

\begin{abstract}
RESUMEN
El uso de sistemas de gestión del aprendizaje (LMS) en las instituciones educativas se ha generalizado en los últimos años, por lo que se hace necesario medir su impacto en los procesos pedagógicos. Con el objetivo de mejorar el uso de la plataforma Moodle como apoyo a los procesos de enseñanza-aprendizaje, en este trabajo se estudia la percepción estudiantil en relación a su utilización en dos asignaturas. Para ello, se utiliza el Modelo de Aceptación Tecnológica (TAM).
\end{abstract}

Se hace un estudio empírico en dos semestres académicos, sobre una muestra de 101 estudiantes, a quienes se les aplicó una encuesta vía web. La información recabada sirvió para realizar análisis estadísticos univariantes y bivariantes y analizar las relaciones estructurales del modelo TAM.

Los resultados obtenidos revelan una actitud moderadamente positiva hacia el uso del aula virtual, reflejan una buena valoración de su facilidad de uso y de su utilidad. Así mismo, se evidencia una buena percepción del diseño instruccional aplicado en las aulas virtuales, pues los estudiantes perciben las actividades implementadas como beneficiosas para su aprendizaje.

Palabras clave: Modelo TAM, tecnología educativa, plataformas educativas, Moodle, diseño instruccional.

\footnotetext{
ABSTRACT

The use of learning management systems (LMS) on educative institutions has been generalized over the last few years, and this is why it is necessary to measure its impact on the pedagogical processes. Aiming to improve the usage of the Moodle platform as a support to the teaching-learning processes, in this paper, the student's perception related to its usage in two subjects is analyzed. For this purpose, the Technological Acceptation Model (TAM) is used. An empiric study over two academic semesters

1 Universidad Nacional de San Agustín de Arequipa. Departamento Académico de Ingeniería de Sistemas e informática. Arequipa, Perú. E-mail: nbedregal@unsa.edu.pe

2 Universidad Nacional de San Agustín de Arequipa. Departamento Académico de Ingeniería de Sistemas e informática. Arequipa, Perú. E-mail: vcornejo@unsa.edu.pe

3 Universidad Nacional de San Agustín de Arequipa. Departamento Académico de Matemáticas. Arequipa, Perú. E-mail: dtupacyupanqui@unsa.deu.pe

4 Universidad Nacional de San Agustín de Arequipa. Maestría en Ingeniería Informática. Arequipa, Perú. E-mail: sidanelia33@gmail.com

* Autor de correspondencia: nbedregal@unsa.edu.pe
} 
is made, over a 101 students' sample, who were interviewed over the web. The collected information was then used to perform uni-variant and bi-variant statistical analysis and perform a structural equation model. The results obtained reveal a moderately positive attitude towards the employment of the virtual classroom, reflecting a good valorization of its simplicity to use and its utility. At the same time, a good perception of the instructional design applied to virtual classrooms is evidenced, as the students perceive the activities implemented as beneficial to their learning.

Keywords: TAM model, educational technology, educational platforms, Moodle, instructional design.

\section{INTRODUCCIÓN}

Las Tecnologías de la Información y Comunicación (TIC) han revolucionado la forma de ver y actuar en el mundo, en particular en el ámbito de la educación han incidido en el diseño de estrategias de masificación y flexibilización, dando origen a la llamada educación virtual $[1,2]$.

Es así que, para cumplir con las políticas de inclusión y de mejora de la calidad, muchas instituciones de educación superior han adoptado el uso de Sistemas de Gestión del Aprendizaje (LMS) -también llamados plataformas virtuales de aprendizaje- en sus procesos pedagógicos. En estas plataformas, las aulas virtuales constituyen espacios de aprendizaje síncronos y asíncronos que incluyen actividades colaborativas, de reflexión y de crítica con el fin de promover aprendizajes significativos [3].

La Universidad Nacional de San Agustín (UNSA) -Arequipa, Perú-, en esta tendencia, en el año 2016 adoptó la plataforma MOODLE como LMS oficial. A partir del año 2017, todos los estudiantes y profesores de la UNSA cuentan con una cuenta de correo de Gmail (con dominio @ unsa.edu.pe) que les permite acceder tanto a la plataforma Moodle institucional como a las funcionalidades de Google.

En este trabajo, tomando como base el modelo TAM, se hace un estudio dirigido a determinar y entender los factores motivacionales que determinan la satisfacción de los estudiantes en relación al uso de la plataforma Moodle, así como analizar su percepción de la utilidad de las herramientas utilizadas y de las actividades implementadas en relación a sus resultados de aprendizaje. El modelo de aceptación de tecnología, conocido por sus siglas en inglés TAM [4] propuesto a finales de los años 80 , modela los factores que hacen que los usuarios acepten y utilicen una tecnología.
Se plantearon como objetivos de este trabajo:

- Describir la percepción de los estudiantes universitarios sobre la facilidad de uso y la utilidad de la plataforma Moodle en los procesos de enseñanza-aprendizaje.

- Determinar los factores que pueden favorecer los procesos de enseñanza-aprendizaje, presenciales, mediante el uso apropiado de la plataforma Moodle.

Con el fin de alcanzar los objetivos planteados, se propusieron las siguientes preguntas de investigación:

- ¿Cómo perciben los estudiantes la facilidad de uso de la plataforma?

- ¿Cómo perciben los estudiantes la utilidad de la plataforma en relación a su aprendizaje?

- ¿Qué cambios realizar en el diseño del aula virtual a fin de mejorar la percepción de los estudiantes?

Como resultado del estudio realizado se concluyó que los estudiantes mantienen una actitud moderadamente positiva hacia el uso del aula virtual implementada en la plataforma Moodle, valoran positivamente su facilidad de uso y su utilidad como facilitadora del proceso de aprendizaje.

Si bien, se confirma la conveniencia de aplicar modelos para medir la aceptación estudiantil hacia las plataformas educativas, también se propone la necesidad de extender el modelo TAM para considerar otras variables externas tales como: apoyo técnico, resistencia al cambio, grado de adecuación, valor didáctico de la herramienta, entre otras.

El aporte de este trabajo radica en considerar el diseño instruccional utilizado en la implementación de las aulas virtuales como variable externa en el modelo TAM, de este modo se está integrando otros elementos significativos en la aceptación de 
las aulas virtuales, integración que puede mejorar el valor explicativo del modelo TAM.

Por otra parte, comparar los resultados asociados a dos asignaturas, posibilita valorar la percepción estudiantil en relación al diseño y utilidad de las estrategias pedagógicas implementadas con apoyo del aula virtual.

\section{MARCO TEÓRICO}

\section{El modelo TAM}

Entre los modelos diseñados para entender la adopción de tecnología, en [5] se afirma que el modelo TAM es uno de los más influyentes, ya que se enfoca en las razones que determinan el rechazo o aceptación de una determinada tecnología de información. TAM relaciona las convicciones, la actitud, la intención y el comportamiento, del usuario, para predecir la aceptación de una TI. En [4] se establece que la utilidad percibida y la facilidad de uso representan las convicciones que llevan a la aceptación de la tecnología y son parte esencial del modelo.

El modelo TAM se estructura en base a cuatro variables que determinan el uso efectivo de la tecnología (Figura 1).

Utilidad Percibida (UP): Grado en el que el usuario estima que el uso de una tecnología podría mejorar su desempeño. La adopción de una tecnología va determinada por la convicción de quien la incorpora de que va a ganar con su uso, ya sea en el plano personal o laboral.

Facilidad de uso Percibida (FU): Grado en el que el usuario cree que el uso de una tecnología estará libre de esfuerzos. Se asocia con el trabajo que se dejará de hacer gracias a la adopción de esa tecnología; luego la pericia en el uso es fundamental.

Actitud hacia el Uso (AU): Sentimiento positivo o negativo relacionado con una conducta. Para adoptar una tecnología resulta indispensable conocer la predisposición del posible usuario.

Intención Conductual hacia el Uso (IC): Grado en el que el usuario formula planes conscientes para desarrollar (o no) una conducta futura. $\mathrm{La}$ intención es proactiva, en el sentido que implica que el usuario se interesará en contar con esa tecnología en la vida diaria.

TAM es una herramienta que se utiliza para conocer las expectativas de un grupo social relacionadas con lo que aporta una tecnología.

\section{La plataforma Moodle como apoyo al modelo educativo presencial}

Moodle fue creado por M. Dougiamas, quien basó su diseño en el enfoque constructivista que sostiene que el conocimiento se construye en la mente del estudiante en lugar de ser transmitido a partir de libros o enseñanzas.

Moodle es una plataforma virtual de aprendizaje que proporciona a educadores, administradores y estudiantes un sistema integrado único, robusto y seguro para crear ambientes de aprendizaje personalizados [6].

Las funcionalidades de Moodle facilitan la implementación de las nuevas tendencias en educación: enfoque basado en competencias, metodologías de aprendizaje activas y evaluación continua, entre otras. Por tanto, es una herramienta poderosa para la formación integral del estudiante.

Fuente: Davis 1989.

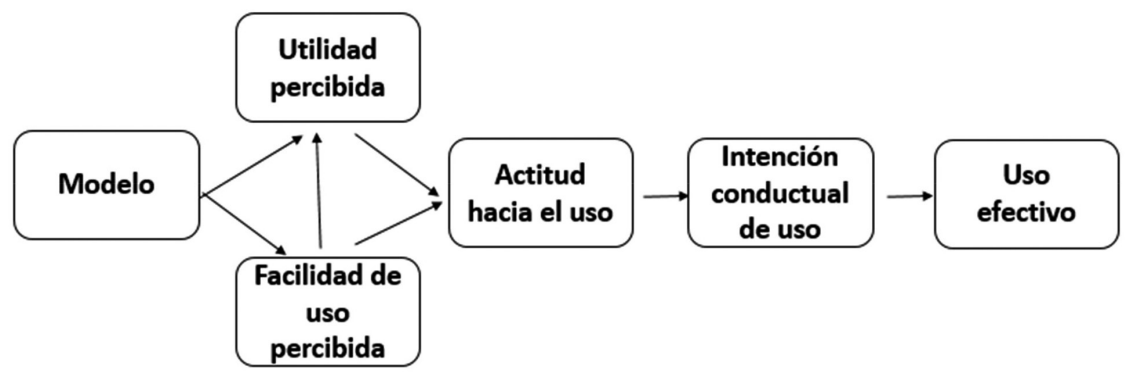

Figura 1. Modelo TAM. 
Moodle permite gestionar una asignatura, compartir contenidos multimedia (apuntes, videos, imágenes, etc.), realizar actividades de evaluación (glosarios, tareas, cuestionarios, encuestas) y actividades comunicativas (foros, mensajería). También, cuenta con la herramienta "calificador", que proporciona cada una de las calificaciones obtenidas por el estudiante, y calcula la calificación promedio.

Las herramientas de un LMS, Moodle en este caso, son fundamentales para crear objetos de aprendizaje o unidades didácticas, e implementar actividades que fomenten el autoaprendizaje y el aprendizaje [7]. El diseño de Moodle está dirigido principalmente al aprendizaje cooperativo.

Moodle es la plataforma de uso libre más utilizada en los modelos educativos semipresenciales, en educación a distancia, clase invertida y diversos proyectos de e-learning en escuelas, universidades, oficinas y otros sectores.

\section{CONTEXTO ACADÉMICO}

\section{Características de las asignaturas}

Las dos asignaturas materia de este estudio se ubican en el Plan de Estudios 2013 de la Escuela Profesional de Ingeniería de Sistemas de la Universidad Nacional de San Agustín, escuela en la que, como apoyo a la enseñanza presencial se ha integrado el uso de la plataforma MOODLE para soportar actividades tales como comunicación síncrona y asíncrona, compartir información, evaluar aprendizajes, recolección y retroalimentación de trabajos prácticos y de laboratorio, evaluaciones, entre otras.

El modelo educativo institucional ha reglamentado la existencia de tres fases durante el semestre académico, en cada fase el docente debe subir al sistema académico dos tipos de calificación: continua y sumativa, por lo que la evaluación de las actividades antes mencionadas se incluye en la "evaluación continua".

La asignatura de Investigación Operativa (IO) se ofrece a los estudiantes durante el sétimo semestre, equivale 4 créditos académicos, cuya distribución en horas lectivas es 2 horas teóricas, 2 horas teórico-prácticas y 2 horas de laboratorio. Para la práctica se incluye el uso de software de aplicación (WinQsb, Excel en combinación con el complemento Solver).
La asignatura Estructuras Discretas II se ofrece en el segundo semestre académico, tienen un peso de tres créditos académicos, equivalentes a 2 horas de teoría y dos horas teórico-prácticas. En el plan de estudios vigente se eliminaron las horas de laboratorio.

\section{Metodología de uso de Moodle}

En la experiencia descrita en este trabajo, se ha utilizado la plataforma Moodle durante dos semestres académicos, en las asignaturas Investigación Operativa (semestre 2017-I) y Estructuras Discretas II (semestre 2017-II).

Para [8] el éxito de un sistema de enseñanzaaprendizaje mediado por tecnología depende directamente del contenido y el grado de integración en el aula.

En el diseño e implementación de las aulas virtuales se debe considerar que, para la mejora de los procesos pedagógicos la tecnología por sí misma no tiene significado, y que es necesaria la intervención oportuna del docente mediante el empleo de estrategias didácticas que promuevan el trabajo activo del estudiante y que estén encaminadas a crear experiencias de aprendizaje que fomenten la atención, motivación y resultados de aprendizaje de los estudiantes.

Es por ello que, dada la variedad de funcionalidades utilizadas para fomentar esas experiencias se eligieron las aulas virtuales de las asignaturas Investigación Operativa y Estructuras discretas II, experiencias que se describen en [3] y [9].

En las aulas virtuales de la plataforma Moodle, las asignaturas se organizaron por unidades de estudio y se separaron algunos bloques específicos:

Bloque Inicio: Se colocó Información de carácter general sobre la asignatura y su desarrollo: información sobre el profesor, sílabo de la signatura y los enlaces a la bibliografía básica, pues debido al peso de los archivos, estos se colocaron en GoogleDrive. Con este bloque se proporcionó a todos los estudiantes la información base para el desarrollo de la asignatura.

Bloque de comunicación/participación: En este bloque, en el tablón de anuncios, se colocaron avisos 
sobre los cambios en horarios, disponibilidad de materiales para su descarga e instrucciones (que iban cambiando de acuerdo al tipo de actividad). Se abrió un foro de comunicación con los estudiantes. De esta manera todos los estudiantes matriculados en la asignatura tuvieron a su alcance la misma información actualizada, aun cuando no hubieran asistido a las sesiones presenciales.

Bloques de contenido: Se implementó un bloque para cada unidad de estudio considerada en el sílabo, en ellos se colocaron tanto contenidos teóricos como prácticos. Se implementaron sub-bloques con:

a) Se colocaron las diapositivas utilizadas en clase, de este modo, los estudiantes tuvieron a su alcance el material educativo antes de su desarrollo en el aula, lo que acercó el modelo presencial a un modelo invertido. Para las actividades de práctica y laboratorio en aula, se publicaron los casos de estudio y ejercicios.

b) Se colocaron materiales de apoyo: videos, lecturas, organizadores de información, referencias online. También se colocaron o enlazaron algunas actividades lúdicas y elementos motivadores.

Bloque de trabajos: Para el envío de tareas, informes, etc.

Bloque de encuestas: Para la recopilación de información relacionada con el desarrollo de la asignatura, para consensuar actividades o fechas, etc.

Boque de Actividad Final: Para apoyar la realización de la actividad de cierre de asignatura cuyas características incluyen trabajo cooperativo, investigación formativa, y desarrollo de las competencias transversales definidas en el sílabo.

\section{DISEÑO DEL ESTUDIO}

Para describir la percepción estudiantil en relación al uso y la utilidad de las funcionalidades de la plataforma Moodle, se eligió el modelo de aceptación tecnológica TAM. Como variable externa se consideró el diseño instruccional del aula virtual, es decir, el tipo de actividades implementadas con apoyo de la plataforma Moodle.

En ambas asignaturas el diseño es similar, lo que difiere es la complejidad de las tareas y actividades. Esta diferenciación se justifica en que los estudiantes matriculados en Estructuras Discretas II generalmente están en el primer año de estudio, mientras que, los matriculados en Investigación operativa van por el cuarto o quinto año de estudio.

\section{Población y muestra}

No existe muestra, se ha trabajado con una población constituida por 101 estudiantes, 53 de los cuales culminaron Investigación Operativa y los restantes 48 culminaron Estructuras Discretas II.

\section{Variables}

En concordancia con los aspectos que evalúa el Modelo de Aceptación de Tecnología, se consideraron las variables:

- MO: Modelo, considerado como el diseño instruccional del aula virtual y los contenidos colocados en ella.

- FU: Facilidad de Uso

Tabla 1. Descripción de los constructos asociados a cada una de las variables.

\begin{tabular}{|c|l|}
\hline Var & \multicolumn{1}{|c|}{ Constructos } \\
\hline MO & Modelo didáctico del aula virtual \\
\hline \multirow{2}{*}{ FU } & FU1. El acceso a los contenidos del aula virtual es sencillo \\
\cline { 2 - 3 } & FU2. Utilizar el material educativo del aula virtual no me genera dificultad \\
\hline \multirow{2}{*}{ UP } & UP1. El material educativo del aula virtual favorece mi aprendizaje \\
\cline { 2 - 3 } & UP2. El aula virtual facilita la entrega de mis tareas. \\
\hline \multirow{2}{*}{ AU } & AU1. Me entusiasman las actividades interactivas del aula virtual. \\
\cline { 2 - 2 } & AU2. Me gusta acceder al material del aula virtual. \\
\hline \multirow{2}{*}{ IC } & IC1. Para mejorar mi aprendizaje participaré más en las actividades del aula virtual \\
\cline { 2 - 3 } & IC2. Si pudiera elegir, seguiría usando el aula virtual \\
\hline
\end{tabular}


- UP: Utilidad Percibida

- AU: Actitud hacia el uso

- IN: Intención conductual

En la Tabla 1 se presentan los constructos relacionadas con las variables TAM.

\section{Instrumento}

El cuestionario es una técnica de recogida de datos cuantitativos que permite una descripción estructurada y general de las variables.

Se construyó un cuestionario dividido en tres secciones:

- En la primera, se recogieron los datos de identificación de los estudiantes (CUI, nombre, edad y asignatura).

- En la segunda, se recogió información asociada a utilidad percibida -en relación con su aprendizaje-, de las actividades implementadas mediante las herramientas de Moodle.

- La tercera sección, recoge la información relativa a las variables del modelo TAM, las preguntas se redactaron tomando como base el trabajo de [10] y, se adaptaron para el entorno cultural en el que se aplicaría la recolección de los datos.

Para la recolección de información, referida a las variables determinantes del modelo, para cada asignatura se realizó una encuesta web a través de la herramienta Google-Forms.

\section{Medición de las variables}

La escala de Likert es una herramienta de medición que permite medir actitudes y conocer el grado de conformidad del encuestado con las afirmaciones que se le propone.

Para recoger la valoración de cada una de las actividades implementadas en el aula virtual en relación al grado en que han favorecido el aprendizaje, se utilizó una escala de Likert con puntuaciones 1 a 5, desde la opción "Totalmente en desacuerdo" hasta "Totalmente de acuerdo", siendo "Indiferente" la posición intermedia (Figura 2).
La codificación de las respuestas fue: 1: Totalmente en desacuerdo, 2: En desacuerdo, 3: Indiferente, 4: De acuerdo y 5: Totalmente de acuerdo.

Como actividades se consideraron la visualización y descarga de documentos, entrega de tareas, evaluaciones, foros, wikis, elaboración y visualización de videos, trabajo cooperativo, encuestas y elaboración y publicación de organizadores de información.

\section{RESULTADOS Y ANÁLISIS}

\section{Valoración del diseño instruccional de las aulas virtuales}

Con la información obtenida en la segunda sección de la encuesta se tuvo un acercamiento a la percepción estudiantil de la utilidad de las actividades en relación a su aprendizaje.

En la Tabla 2 se consigna la cantidad de estudiantes que eligieron cada puntuación, así mismo, la media aritmética y la desviación estándar de las puntuaciones obtenidas para cada actividad implementada en el aula virtual de las asignaturas.

Como se aprecia en la Tabla 2, las actividades obtuvieron puntuaciones promedio satisfactorias cercanas a los 4 puntos, por lo que se puede inferir que los estudiantes están de acuerdo con las actividades planteadas. En ninguno de los casos se llega a bajar hasta el nivel de indiferencia.

Las Figuras 3 y 4, muestran las valoraciones (en porcentaje) otorgadas por los estudiantes a las actividades realizadas a través del aula virtual en el desarrollo de Investigación Operativa y Estructuras Discretas II.

Se observa que la actividad más valorada en ambos casos es el Trabajo en Equipo, para gestionar esta actividad se utilizaron diversas funcionalidades de Moodle: correo (para la comunicación docenteestudiantes), tareas (para recoger avances y dar retroalimentación), etiquetas (para dar información resumida y consensuada), cuestionario (para evaluar y recoger información), etc.

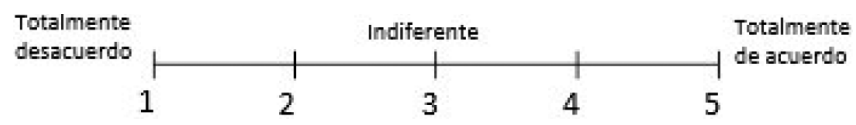

Figura 2. Valores en la escala de Likert. 
Tabla 2. Puntuaciones y medidas descriptivas de la percepción de la utilidad por actividad.

\begin{tabular}{|l|c|c|c|c|c|c|c|c|c|c|c|c|c|c|}
\cline { 2 - 16 } \multicolumn{1}{c|}{} & \multicolumn{4}{|c|}{ Investigación operativa } & \multicolumn{4}{c|}{ Estructuras discretas 2 } \\
\cline { 2 - 16 } & $\mathbf{1}$ & $\mathbf{2}$ & $\mathbf{3}$ & $\mathbf{4}$ & $\mathbf{5}$ & $\boldsymbol{\mu}$ & $\sigma$ & $\mathbf{1}$ & $\mathbf{2}$ & $\mathbf{3}$ & $\mathbf{4}$ & $\mathbf{5}$ & $\boldsymbol{\mu}$ & $\sigma$ \\
\hline Documentos & 2 & 2 & 12 & 25 & 13 & 3,83 & 0,96 & 2 & 2 & 11 & 21 & 12 & 3,81 & 0,99 \\
\hline Tareas & 0 & 2 & 8 & 26 & 18 & 4,11 & 0,79 & 0 & 1 & 6 & 24 & 17 & 4,19 & 0,73 \\
\hline Evaluaciones & 1 & 3 & 8 & 28 & 14 & 3,94 & 0,89 & 0 & 1 & 7 & 22 & 18 & 4,19 & 0,75 \\
\hline Foro & 3 & 3 & 20 & 18 & 10 & 3,54 & 1,03 & 0 & 2 & 15 & 18 & 13 & 3,88 & 0,86 \\
\hline Wiki & 0 & 1 & 8 & 21 & 24 & 4,26 & 0,77 & 0 & 2 & 8 & 22 & 16 & 4,08 & 0,81 \\
\hline Videos & 1 & 1 & 10 & 21 & 21 & 4,11 & 0,9 & 0 & 0 & 7 & 22 & 19 & 4,25 & 0,69 \\
\hline Trab. Equipo & 0 & 0 & 6 & 22 & 26 & 4,37 & 0,67 & 0 & 0 & 2 & 18 & 28 & 4,54 & 0,58 \\
\hline Organizadores & 0 & 2 & 8 & 20 & 24 & 4,22 & 0,83 & 0 & 1 & 5 & 20 & 22 & 4,31 & 0,74 \\
\hline
\end{tabular}

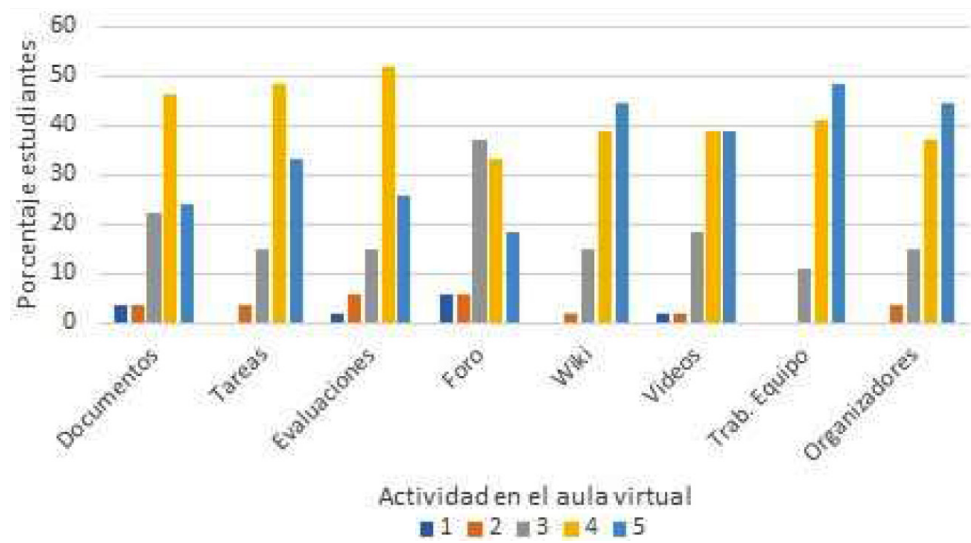

Figura 3. Valoración de las actividades en el aula virtual de Investigación Operativa.

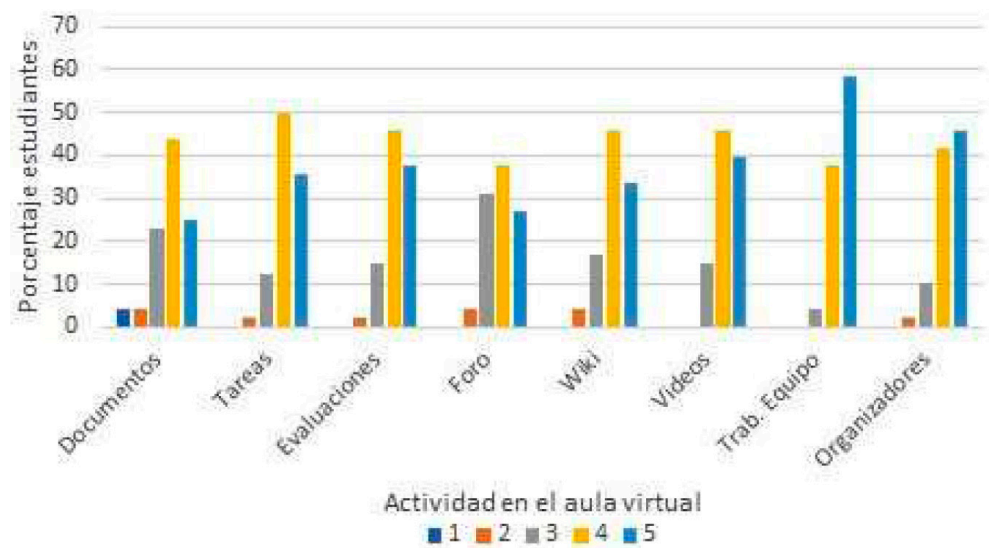

Figura 4. Valoración de las actividades en el aula virtual de Estructuras Discretas II.

Es necesario destacar que, en Investigación Operativa, la actividad cooperativa consistió en la creación de un banco de preguntas para las evaluaciones vía aula virtual; preguntas que fueron elaboradas por los estudiantes [9]. En Estructuras Discretas II, por tratarse de estudiantes de segundo semestre, la actividad cooperativa fue más simple y se realizó con la intervención del profesor. 
Los organizadores de información (implementados con la funcionalidad etiquetas) son mayormente aceptados en Estructuras Discretas 2, lo que se explica por la naturaleza de la asignatura que demanda mayor trabajo de abstracción en estudiantes de segundo semestre.

Las evaluaciones (implementadas con la funcionalidad cuestionarios) son significativas en ambas asignaturas, pues contribuyeron a evaluar el proceso y no solo los resultados del aprendizaje. Sin embargo, con los estudiantes de Investigación Operativa no fue fácil consensuar la hora de la evaluación en línea, pues varios estudiantes trabajan o tenían problemas con la conexión a internet.

La actividad en los foros obtuvo bajas valoraciones y mayor variabilidad, lo que podría explicarse por la baja participación de los estudiantes; situación que se puede corregir motivando al estudiante a expresar sus dudas y cuestionamientos a través del foro, y dando lugar a la absolución de los mismos tanto por parte del profesor como de los mismos estudiantes.

La actividad Wiki fue muy valorada en Investigación Operativa, pues se utilizó para que los mismos estudiantes propusieran ejemplos de modelación, lo que les permitió identificar situaciones tipo.

La descarga de documentos fue la actividad menos valorada como favorecedora del aprendizaje.

\section{Valoración del modelo TAM}

Para evaluar la consistencia interna del instrumento, se ha utilizado el coeficiente $\alpha$ de Cronbach, que permite estimar la fiabilidad de un instrumento de medida a través de un conjunto de ítems que se espera que midan el mismo constructo o dimensión teórica.

Como criterio general, en [11] se dan las siguientes recomendaciones para evaluar los coeficientes de Alfa de Cronbach:

- Coeficiente alfa $>0,9$ es excelente.

- Coeficiente alfa $>0,8$ es bueno.

- Coeficiente alfa $>0,7$ es aceptable.

- Coeficiente alfa $>0,6$ es cuestionable.

- Coeficiente alfa $>0,5$ es pobre.

- Coeficiente alfa $<0,5$ es inaceptable.

El coeficiente debe obtenerse con los datos de cada muestra, así se garantiza la medición fiable del constructo en la muestra concreta de investigación. En la Tabla 3, se detallan los coeficientes Alfa de Cronbach por cada constructo y por cada asignatura.

Tabla 3. Prueba de confiabilidad de las variables del Modelo TAM.

\begin{tabular}{|l|c|c|}
\cline { 2 - 3 } \multicolumn{1}{c|}{} & \multicolumn{2}{c|}{ Alfa de Cronbach } \\
\hline Variable & $\begin{array}{c}\text { Investigación } \\
\text { operativa }\end{array}$ & $\begin{array}{c}\text { Estructuras } \\
\text { Discretas II }\end{array}$ \\
\hline Modelo & 0,88 & 0,86 \\
\hline Facilidad de uso & 0,83 & 0,82 \\
\hline Utilidad Percibida & 0,75 & 0,73 \\
\hline Actitud hacia el uso & 0,80 & 0,80 \\
\hline Intención conductual & 0,85 & 0,86 \\
\hline
\end{tabular}

Como los resultados indican una buena consistencia interna de los ítems analizados, con la información

Tabla 4. Estadísticos descriptivos por constructo.

\begin{tabular}{|l|c|c|c|c|}
\cline { 2 - 5 } \multicolumn{1}{c|}{} & \multicolumn{2}{c|}{ Investigación operativa } & \multicolumn{2}{c|}{ Estructuras Discretas 2 } \\
\hline Constructo & Media aritmética & Desviación estándar & Media aritmética & Desviación estándar \\
\hline MO & 4,07 & 0,36 & 4,12 & 0,32 \\
\hline FU1 & 4,13 & 0,64 & 4,10 & 0,58 \\
\hline FU2 & 3,94 & 0,83 & 3,95 & 0,73 \\
\hline UP1 & 4,09 & 0,62 & 4,13 & 0,63 \\
\hline UP2 & 4,30 & 0,71 & 4,29 & 0,64 \\
\hline AU1 & 4,11 & 0,63 & 4,19 & 0,52 \\
\hline AU2 & 3,92 & 0,74 & 3,96 & 0,64 \\
\hline IC1 & 4,17 & 0,54 & 4,25 & 0,55 \\
\hline IC2 & 3,74 & 0,80 & 3,81 & 0,72 \\
\hline
\end{tabular}


obtenida de la tercera sección de la encuesta se pudo valorar la percepción estudiantil en relación a las variables del modelo TAM.

La Tabla 4 muestra los resultados descriptivos de cada uno de los constructos para las dos asignaturas en estudio.

Las Figuras 5 y 6 muestran el porcentaje de estudiantes que optó por una puntuación específica de la escala de Likert para cada uno de los constructos.

De estos resultados se infiere que en ambas asignaturas los estudiantes percibieron que el acceso al aula virtual y a su contenido (FU1 y FU2) es sencillo. En este punto, es conveniente recordar que son estudiantes de la Escuela de Ingeniería de Sistemas, por lo que un resultado diferente no resultaría lógico.

Si bien las puntuaciones promedio para la utilidad percibida superan los 4 puntos, la facilidad en la entrega de tareas (UP2) supera ligeramente la puntuación de la utilidad percibida en relación a favorecer el aprendizaje.

En la actitud hacia el uso del aula virtual (AU2), se obtuvo menor valoración lo que posiblemente se debe a la mayor cantidad de trabajo que deben realizar en esta modalidad combinada

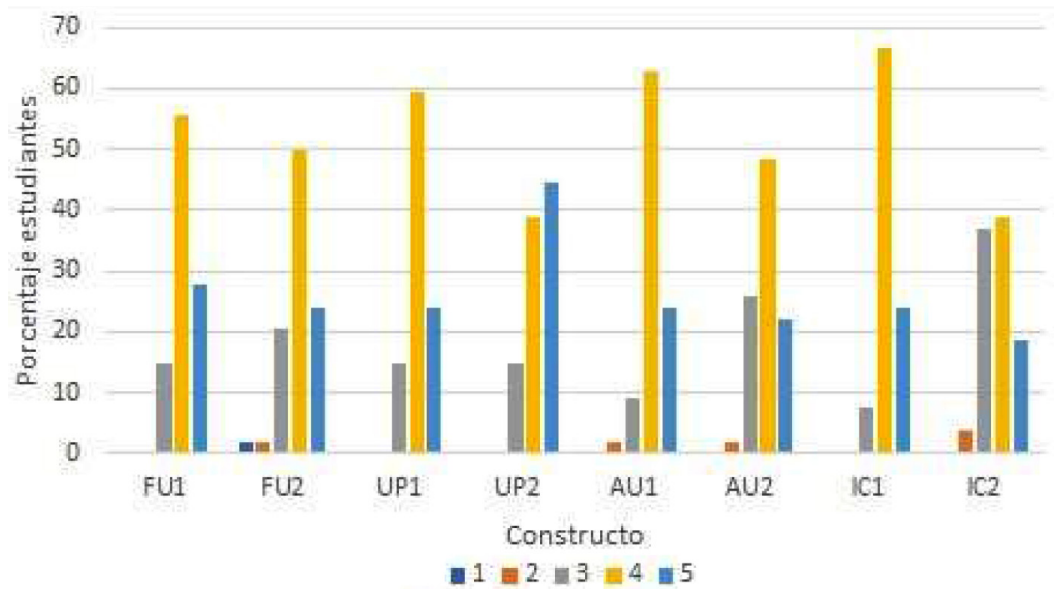

Figura 5. Porcentaje de estudiantes que escogieron una puntuación por constructo: Investigación Operativa.

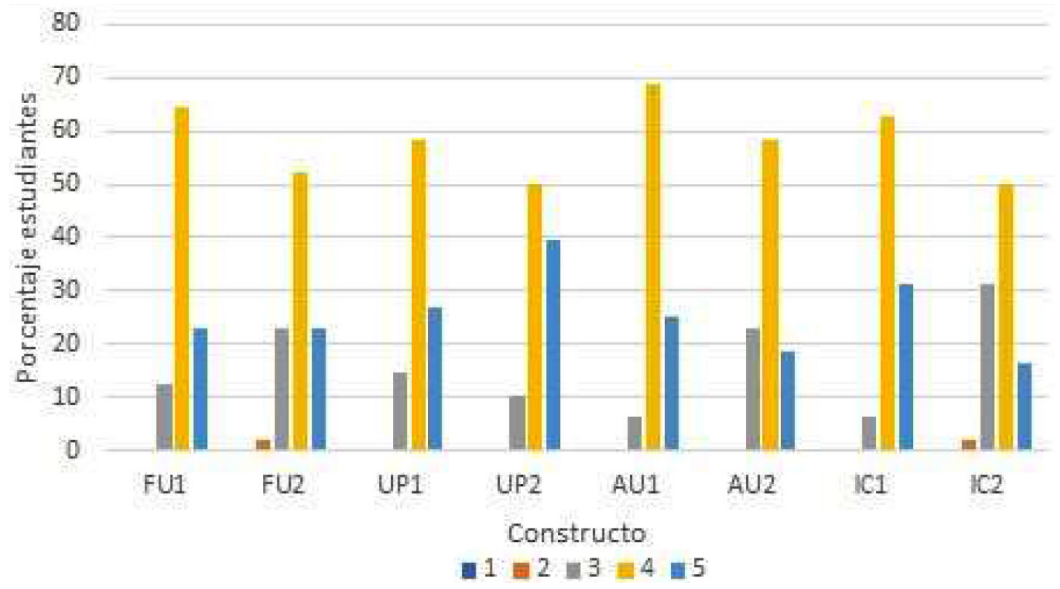

Figura 6. Porcentaje de estudiantes que escogieron una puntuación por constructo: Estructuras Discretas II. 
(presencial-virtual) comparada al trabajo en una asignatura con modalidad netamente presencial.

Analizando los resultados de la actitud hacia el uso y la intención de uso (IC1) se puede decir que el estudiante percibe la utilidad del aula virtual para favorecer su aprendizaje, sin embargo, aún le causa resistencia la cantidad de trabajo extra que esto le significa.

Para analizar la relación entre las variables del modelo TAM, se midió la correlación existente entre las variables relacionadas en el modelo (Tabla 5).

Tabla 5. Coeficiente de correlación de Pearson de las variables TAM.

\begin{tabular}{|l|c|c|}
\hline Variables & $\begin{array}{c}\text { Investigación } \\
\text { operativa }\end{array}$ & $\begin{array}{c}\text { Estructuras } \\
\text { Discretas 2 }\end{array}$ \\
\hline $\mathrm{MO}$ - FU & 0,75 & 0,70 \\
\hline $\mathrm{MO}-\mathrm{UP}$ & 0,74 & 0,76 \\
\hline $\mathrm{FU}-\mathrm{UP}$ & 0,73 & 0,74 \\
\hline FU - AU & 0,69 & 0,61 \\
\hline UP - AU & 0,71 & 0,62 \\
\hline AU - IC & 0,62 & 0,54 \\
\hline
\end{tabular}

Las Figuras 7 y 8 muestran, en la gráfica del Modelo TAM, los índices de correlación obtenidos entre

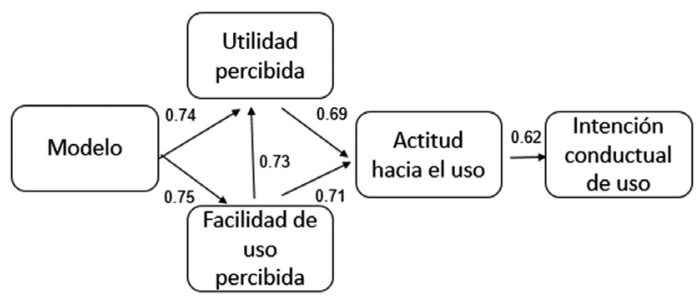

Figura 7. Correlación entre variables: Investigación Operativa.

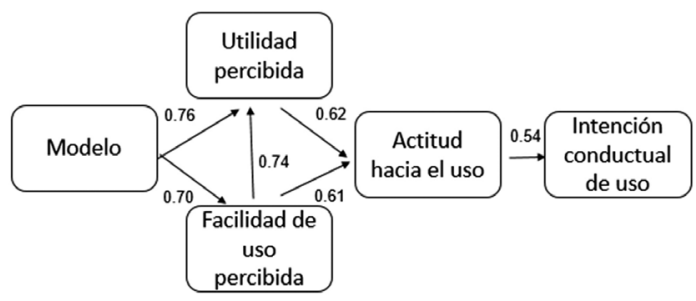

Figura 8. Correlación entre variables: Estructuras Discretas II. las variables del modelo correspondiente a cada asignatura.

Del análisis del valor de los coeficientes de correlación obtenidos, se desprende que no existen problemas de colinealidad, por lo que las variables que interactúan deben mantenerse en el modelo, valores de correlación superiores a $r=0,85$ pueden señalar problemas potenciales [12].

De haberse encontrado dos variables altamente correlacionadas, se habría tenido que eliminar una de ellas.

Se observa que las variables Modelo y Facilidad de Uso, explican en buena medida la utilidad percibida por los estudiantes. El bajo resultado obtenido por la variable Actitud hacia el uso sobre la intención conductual de seguir utilizando la plataforma se puede explicar por la misma idiosincrasia del estudiante, sobre todo en los primeros años de estudio, que le lleva considerar actividades de menor esfuerzo.

Análogamente se observa que, la Facilidad de Uso percibida por el estudiante tiene una influencia importante sobre la Actitud hacia el Uso.

\section{CONCLUSIONES}

Los resultados obtenidos, en este trabajo, revelan una actitud moderadamente positiva de los estudiantes hacia el uso del aula virtual implementada en la plataforma Moodle, reflejan una buena valoración de la utilidad y facilidad de uso del aula virtual. Así mismo, se evidencia una buena percepción del diseño instruccional aplicado en las aulas virtuales, pues los estudiantes perciben las actividades implementadas como beneficiosas para su aprendizaje.

En base al trabajo realizado, se concluye que las herramientas de la plataforma en general se perciben como útiles y fáciles de utilizar, esta percepción puede verse sesgada por la especialidad de estudio. Tendrían que investigarse los resultados con estudiantes de otras especialidades, al respecto, en [13] se reportan problemas de navegación y la necesidad de capacitar previamente a los estudiantes en el uso de la plataforma.

Las relaciones causales entre las variables consideradas están bien explicadas, de modo tal que, 
el estudio prueba de la conveniencia de aplicar modelos para medir la aceptación estudiantil hacia las plataformas educativas.

El modelo de aceptación tecnológica (TAM), no considera si la aceptación de la tecnología incide o no en el incremento del rendimiento del usuario, [14] y [15] plantean que el hecho de que una tecnología sea usada, no implica mejoras en el rendimiento del usuario.

Para comprender la eficacia de utilizar un LMS en el contexto educativo universitario, es necesario agregar al modelo TAM otras variables externas que influyen directamente en la utilidad y en la facilidad de uso percibidas: apoyo técnico, resistencia al cambio, grado de adecuación, valor didáctico de la herramienta, entre otras. En el contexto universitario, en [16] se utilizó el modelo TAM y se encontró que era una buena herramienta teórica para comprender la aceptación de los usuarios de e-learning, al incluir autoeficacia y norma subjetiva en el uso de e-learning se concluyó que eran los constructos más importantes en la explicación conductual del modelo.

Por tanto, se puede concluir que los estudiantes presentan una buena disposición a utilizar el aula virtual implementada en la plataforma Moodle, siempre y cuando encuentren que las actividades a realizar favorecen su aprendizaje y no encuentren dificultades en su utilización. Es necesario entonces, crear un balance entre el esfuerzo invertido y los beneficios que les reportaría.

\section{RECOMENDACIONES}

Es necesario potenciar la implementación de actividades interactivas en el aula virtual, por ejemplo, la actividad foro de tutoría virtual, actividad que facilitaría la solución de dudas mediante la intervención del profesor o de otros estudiantes.

Los resultados obtenidos refuerzan el conocimiento existente respecto a algunas de las ventajas del uso de plataformas virtuales en los procesos de enseñanza-aprendizaje, no obstante, es indispensable realizar estudios más profundos a fin de determinar su incidencia en los resultados de aprendizaje y en la calidad de los procesos educativos.

\section{RECONOCIMIENTOS}

Este trabajo se realizó con el apoyo de nuestra casa de estudios, la Universidad Nacional de San Agustín de Arequipa, en la que el Vicerrectorado Académico ha puesto en marcha un plan de capacitación docente en Didáctica Superior e integración de TIC al proceso educativo y el Vicerrectorado de Investigación canaliza los recursos provenientes del canon minero y convoca a un conjunto de esquemas financieros concursables que apoyan el financiamiento de los trabajos de investigación.

\section{REFERENCIAS}

[1] S. Mohammadyari and H. Singh. "Understanding the effect of e-learning on individual performance: The role of digital literacy". Computers \& Education. Vol. 82, pp. 11-25. 2015.

[2] M. Paechter y B. Maier. "Online or facetoface? Students' experiences and preferences in e-learning". The internet and higher education. Vol. 13 No 4, pp. 292-297. 2010.

[3] N. Bedregal y D. Tupacyupanqui. "Integración de metodologías activas y aula virtual en los procesos enseñanza-aprendizaje de Matemática Discreta". 16th LACCEI International Multi-Conference for Engineering, Education, and Technology: Innovation in Education and Inclusion". 19-21 July 2018, Lima. DOI: 10.18687/ LACCEI2018.1.1.81

[4] F. Davis, R. Bagozzi and P. Warshaw. "User acceptance of computer technology: A comparison of two theoretical models". Management Science. Vol. 35, pp. 982-1003. 1989.

[5] M. Dias and R. Zwicker. "A evolucão do modelo de aceitacão de tecnologia de Davis: uma análise do original e variacões de 1985 até 2003". Encontro do CLADEA - Asamblea Anual del Consejo Latinoamericano de Escuelas de Administración, Lima. 2003.

[6] Moodle. 28 de enero de 2016. Moodle. Obtenido de https://docs.moodle.org/all/es/ Acerca_de_Moodle

[7] I. Ros. "Moodle, la plataforma para la enseñanza y organización escolar". Ikastorratza, e- Revista de Didáctica (2008). Recuperado de http:// www.ehu.es/ikastorratza/2_alea/moodle.pdf 
[8] C. Riemer. "Autonomía y motivación”. Acta del simposio Bremer. 2009.

[9] N. Bedregal. "Aprendizaje cooperativo usando Moodle como recurso de apoyo: Propuesta de evaluación continua en la asignatura Investigación Operativa". XVIII Congreso Chileno de Educación en Computación 2017Jornadas Chilenas de Computación 2017. Arica, Chile. DOI: 10.1109/SCCC.2017.8405131

[10] F. Davis. "Perceived usefulness, perceived ease of use, and user acceptance of information technology". MIS Quarterly. Vol. $13 \mathrm{~N}^{\mathrm{o}} 3$, pp. 319-340. 1989.

[11] D. George and P. Mallery. "SPSS for Windows step by step: A Simple Guide and Reference". 11.0 Update ( $4^{\mathrm{a}}$ ed.). Boston: Allyn \& Bacon. 2003.

[12] R. Kline. "Methodology in the social sciences. Principles and practice of structural equation modeling". 2nd ed. New York, NY, US: Guilford Press. 2005.

[13] S. Tee and T. Wook. "User testing for Moodle application". International Journal of Software Engineering and its Applications. Vol. $7 \mathrm{~N}^{\circ} 5$ : pp. 243-252. 2013.

[14] D. Goodhue and R. Thompson. "Tasktechnology fit and individual performance". MIS quarterly. Vol. 19, pp. 213-236. 1995.

[15] Y. Lee. "The technology acceptance model: Past, present, and future". Communications of the Association for Information Systems. Vol. 12, p. 780. 2003.

[16] S. Park. "Un análisis del modelo de aceptación de la tecnología para la comprensión de la intención conductual de los estudiantes universitarios en usar el aprendizaje electrónico". Educational Technology \& Society. Vol. 12 No 3, pp. 150-162. 2009. 